\title{
Water Wave Interaction by Dual Cylindrical Cylinders with Partial Porous Area
}

\author{
Min-Su Park, Youn-Ju Jeong, and Young-Jun You
}

\begin{abstract}
In the present study, the 3D numerical analysis method is developed with Eigenfunction expansion method to evaluate the wave forces acting on the array of dual cylindrical cylinders with partial porous area, which consist of an impermeable inner cylinder and a porous outer cylinder. The wave forces and water wave interaction on dual cylindrical cylinders with partial porous area are presented for various porosity depths, and the comparison between the impermeable cylinder and the permeable cylinder is made to examine the effects of porosity. From these results, the present method is very useful to evaluate the wave force and water wave interaction acting on the array of dual cylindrical cylinders with partial porous area.
\end{abstract}

Index Terms-Eigenfunction expansion method, dual cylindrical cylinder, partial porous area, water wave interaction, wave force.

\section{INTRODUCTION}

There is a large variety of offshore structures in ocean. An offshore structure is generally subjected to very different situations from a shore structure because it is subjected to severe environmental conditions such as wave forces, wind forces and current forces etc. To perform the reliable design of an offshore structure, it is very important to exactly evaluate the wave forces acting on the offshore structure.

It is well recognized that the wave diffraction problem about a vertical circular cylinder carried out by MacCamy and Fuchs [1] is a typical problem with exact analytical solution in ocean engineering. As regarding shallow water wave diffraction around a vertical cylinder, Isaacson [2], [3] was among the pioneers to derive analytical solutions. Under the assumptions of potential flow and linear wave theory, a semi-analytical solution is obtained by an Eigenfunction expansion approach first proposed for impermeable cylinders (Spring and Monkmeyer [4]), and latter simplified by Linton and Evans [5] for $N$ bottom-mounted circular cylinders. Kagemoto and Yue [6] have developed another solution that is formally exact within the context of the linear theory. In the case where the cylinder spacing is large relative to the incident wave length, approximate techniques may reasonably be used to quantify the hydrodynamic interactions between the members of multi-column structures. A popular approach, based on the wide-spacing assumption is the so-called modified plane-wave approach first developed by McIver and Evans [7], and later used in a number of

Manuscript received February 15, 2016; revised March 30, 2016.

Min-Su Park, Youn-Ju Jeong, and Young-Jun You are with the Structural Engineering Research Institute, Korea Institute of Civil Engineering and Building Technology, Goyang-Si, South Korea (e-mail: mspark@kict.re.kr, yjjeong@kict.re.kr, yjyou@kict.re.kr). applications by McIver, Williams and Demirbilek [8], Williams and Abul-Azm [9], and Williams and Rangappa [10]. All of the above studies, however, assume that the cylinders are impermeable.

In case of porous cylinders, the wave motions in the exterior and all interior fluid regions are expressed by Williams and Li [11], [12]. Wang and Ren [13] were the earliest to study the wave interaction with a concentric surface piercing porous outer cylinder protecting an impermeable inner cylinder. It was reported that the outer porous cylinder is significantly effective to reduce the hydrodynamic force and wave run-up on the inner cylinder compared to be exposed to direct wave impact. Wang and his research group (Wang et al. [14], Wang and Jiang [15], Jiang and Wang [16], Wan and Ren [17], [18]) carried out a systematic numerical analysis of solitary and cnoidal waves interacting with a vertical cylinder or cylinder arrays.

In the present study, the 3D numerical analysis method is developed with Eigenfunction expansion method which is expressed by Williams and Li [12], and Wang and Len [13]. The analysis method could be applied for the wave force evaluation to the array of dual cylindrical cylinders with partial porous area, which consist of an impermeable inner cylinder and a porous outer cylinder. Firstly, the results obtained from the developed numerical method are compared with ones such as Linton and Evans [5], and Wang and Ren [13] to verify the developed numerical analysis method. Moreover, the wave forces and water wave interaction on dual cylindrical cylinders with partial porous area are presented for various porosity depths, and the comparison between the impermeable cylinder and the permeable cylinder is made to examine the effects of porosity. From these results, the present method is very useful to evaluate the wave force acting on the array of dual circular cylinders with partial porous area. Moreover the interaction effects between wave and cylinders are closely related to the diameter of cylinder and the spacing between cylinders. In other words, the porosity of cylinders is remarkably effective to reduce the interaction effects.

\section{FORMULATION}

It is assumed that the computational fluid domain is inviscid and incompressible, and its motion is irrotational. An arbitrary array of $N$ dual cylindrical cylinders with partial porous area is situated in water of uniform depth $d$ and the draughts of each permeable and impermeable area of dual cylindrical cylinders with partial porous area are $h$ and $c$, respectively. The outer and the inner radius of the $j$ th dual cylindrical cylinder are $a_{j}$ and $b_{j}$, respectively. Also, the 
global Cartesian coordinate system $(x, y, z)$ is defined with an origin located on the sea bed with the $z$-axis directed vertically upwards. The center of each dual cylindrical cylinder at $\left(x_{j}, y_{j}\right)$ is taken as the origin of a local polar coordinate system $\left(r_{j}, \theta_{j}\right)$, where $\theta_{j}$ is measured counterclockwise from the positive $x$-axis. The center of the $l$ th dual cylindrical cylinder has a polar coordinate $\left(R_{j l}, \alpha_{j l}\right)$ relative to the $j$ th dual cylindrical cylinder. The coordinate relationship between the $j$ th and $l$ th dual cylindrical cylinder is shown in Fig. 1. Moreover, the fluid domain is divided into two regions: region 1 which is interior to the dual cylindrical cylinder $\left(b_{j} \leq r_{j} \leq a_{j}, d-h \leq z \leq d\right)$ and region 2 which is exterior to the dual cylindrical cylinder and extends to infinity in the horizontal plane $\left(r_{j} \geq a_{j}, 0 \leq z \leq d\right)$.
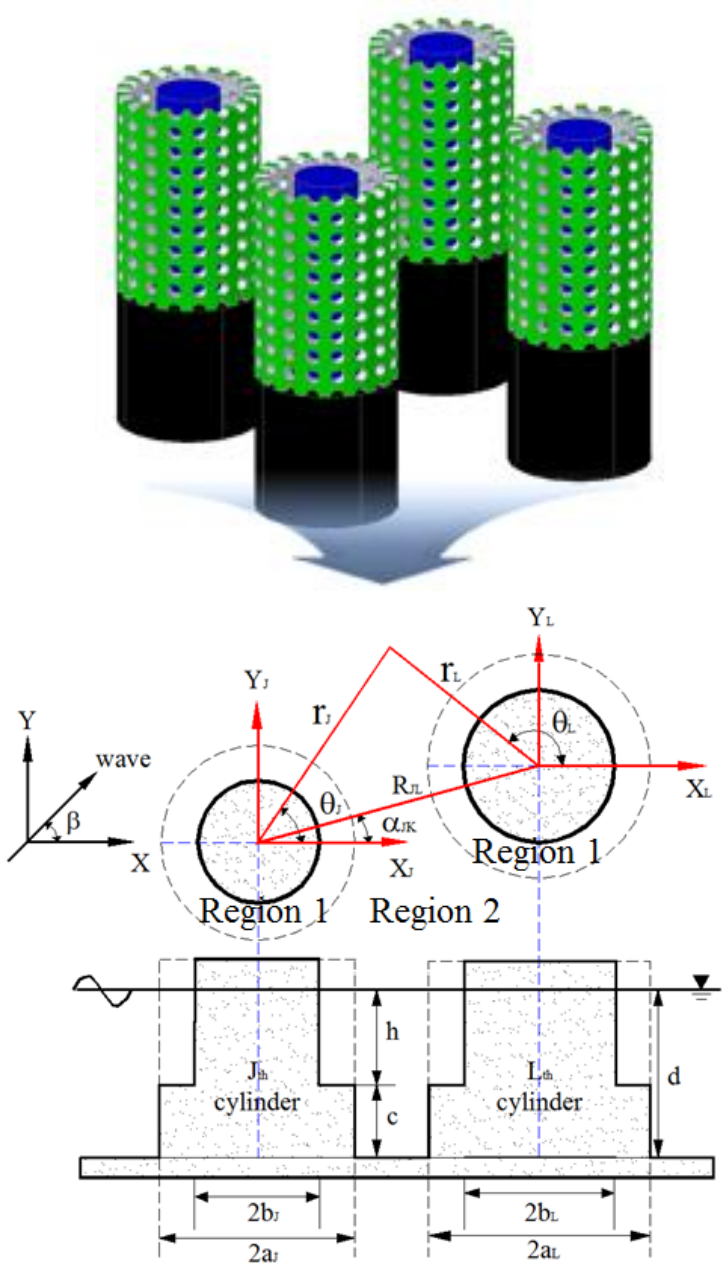

Fig. 1. Coordinate system for an array of dual cylindrical cylinders with partial porous area.

The array of dual cylindrical cylinders is subjected to a train of regular waves of height $H$ and angular frequency $\omega$ propagating at an angle $\beta$ to the positive $x$-axis. The velocity potential of the computational domain can be written as

$$
\Phi(x, y, z, t)=\operatorname{Re}\left\lfloor-\{i g H /(2 \omega)\} \phi(x, y, z) e^{-i \omega t}\right\rfloor
$$

where $\operatorname{Re}[$ ] denotes the real part of a complex velocity potential $\Phi$, and $g$ is the gravitational acceleration.

As a governing equation, the Laplace equation is satisfied for the entire fluid domain of the present boundary value problem:

$$
\nabla^{2} \phi=0
$$

For solving the governing equation, the following boundary conditions for the free surface (Eq. (3)), bottom of region 1 (Eq. (4)), vertical wall of upper and lower area (Eq. (5)), flat rigid sea bottom (Eq. (6)), and the Sommerfeld radiation boundaries (Eq. (7)) can be given, respectively:

$$
\begin{aligned}
& \frac{\partial \phi}{\partial z}-\frac{\omega^{2}}{g} \phi=0 \quad \text { on } \quad z=d \\
& \frac{\partial \phi}{\partial z}=0 \quad \text { on } \quad z=d-h, \quad b_{j} \leq r \leq a_{j} \\
& \frac{\partial \phi}{\partial r_{j}}=0 \quad \text { on } \quad r=b_{j}, \quad(d-h) \leq z \leq d \\
& \frac{\partial \phi}{\partial z}=0 \quad \text { on } \quad r=a_{j}, \quad 0 \leq z \leq(d-h) \\
& \lim _{r \rightarrow \infty} \sqrt{r}\left\lfloor\frac{\partial}{\partial r}\left(\phi_{2}-\phi_{i n}\right)-i k\left(\phi_{2}-\phi_{i n}\right)\right\rfloor=0
\end{aligned}
$$

where $k$ is the incident wave number related to the wave frequency through the dispersion relation $\omega^{2}=g k \tanh k h$, and $d$ is the water depth. $\phi_{2}$ and $\phi_{i n}$ denote the total velocity potential in region 2 and the incident wave potential, respectively.

The wave potential in the interior region(1) of the $j$ th cylinder, which satisfies the appropriate free surface and structural boundary conditions, can be expressed by the following Eigenfunction expansion,

$$
\phi_{1}^{j}=\sum_{n=-\infty}^{n=\infty}\left\lfloor A_{n}^{j} J_{n}\left(k_{0} r_{j}\right)-\frac{J_{n}^{\prime}\left(k_{0} b_{j}\right)}{Y_{n}^{\prime}\left(k_{0} b_{j}\right)} A_{n}^{j} Y_{n}\left(k_{0} r_{j}\right)\right\rfloor \times \frac{\cosh \left\{k_{0}(z-d+h)\right\}}{\cosh \left(k_{0} h\right)} e^{i n \theta_{j}}
$$

in which $J_{n}$ and $Y_{n}$ denotes the Bessel function of the first and the second kind of order $n$, and $J_{n}^{\prime}$ and $Y_{n}^{\prime}$ are the first derivatives of the Bessel function, respectively. $A_{n}{ }^{j}$ is the unknown complex potential coefficient. A new wave number $k_{0}$ is introduced, which satisfies the dispersion relation $\omega^{2}=g k_{0} \tanh k_{0} h$, where $h$ denote local water depth in the interior region 1.

The incident wave potential in the $j$ th local polar coordinate system can be expressed using Jacobi-Anger expansion of Bessel function as follows,

$$
\phi_{i n}^{j}=\frac{\cosh k z}{\cosh k d} T_{j} \sum_{n \rightarrow-\infty}^{\infty} J_{n}\left(k r_{j}\right) e^{i n\left(\pi / 2+\theta_{j}-\beta\right)}
$$

where $T_{j}=e^{i k\left(x_{j} \cos \beta+y_{j} \sin \beta\right)}$ is a phase factor associated with the cylinder $j$ from the global origin.

The wave potential in the exterior region (2), which is expressed by using Graf's addition theorem for the Bessel functions [19] and satisfies the Helmholtz equation, can be expressed by the following Eigenfunction expansion, 


$$
\begin{aligned}
& \phi_{2}^{j}\left(r_{j}, \theta_{j}\right)= \\
& \sum_{n=-\infty}^{n=\infty}\left[\begin{array}{l}
e^{i k\left(x_{j} \cos \beta+y_{j} \sin \beta\right)} J_{n}\left(k r_{j}\right) e^{i n(\pi / 2-\beta)}+C_{n}^{j} \frac{H_{n}\left(k r_{j}\right)}{H_{n}^{\prime}\left(k a_{j}\right)} \\
+\sum_{\substack{l=1 \\
l \neq j}}^{N} \sum_{m=-\infty}^{m=\infty} C_{m}^{l} \frac{a_{l} J_{n}\left(k r_{j}\right)}{a_{j} H_{m}{ }^{\prime}\left(k a_{l}\right)} H_{m-n}\left(k R_{l j}\right) e^{i(m-n) \alpha_{l j}}
\end{array}\right] \times \frac{\cosh k z}{\cosh k d} e^{i n \theta_{j}}
\end{aligned}
$$

The right-hand side of Eq. (10) represents the incident wave upon the $j$ th cylinder, the scattered wave produced by the $j$ th cylinder, and the re-scattered wave generated by the adjacent cylinder $l$, respectively. $C_{n}^{j}$ is the unknown complex potential coefficient. $H_{n}$ is the Hankel function of the first kind of order $n$, and $H_{n}^{\prime}$ is the first derivatives of the Hankel function, respectively.

The fluid flow passing through the porous surface of dual cylindrical cylinder is assumed to obey Darcy's law. Therefore it can be written as follows [12],

$$
\frac{\partial \phi_{1}^{(j)}}{\partial r}=\frac{\gamma}{\mu} \rho i \omega\left[\phi_{1}^{(j)}-\phi_{2}^{(j)}\right] \text { on } r_{j}=a_{j}, j=1,2,3, \ldots, N
$$

where $\mu$ is the coefficient of dynamic viscosity, $\gamma$ is a material constant having the dimension of length and $\rho$ is the fluid density, respectively. Subsequently, the porosity of the dual cylindrical cylinder will be characterized by the dimensionless parameter, $G$. The body boundary condition on the porous surface of dual cylindrical cylinder can be expressed with the $G$.

$$
\frac{\partial \phi_{1}^{(j)}}{\partial r}=i k_{0} G\left[\phi_{1}^{(j)}-\phi_{2}^{(j)}\right] \text {, Here } G=\frac{\rho \omega \gamma}{\mu k_{0}}
$$

In addition to applying the body boundary conditions associated with the free surface conditions, the present boundary value problem must satisfy the matching conditions at the interface between the regions, which are given by

$$
\begin{aligned}
& \phi_{1}^{j}=\phi_{2}^{j} \quad \text { on } \quad r_{j}=a_{j}, d-h \leq z \leq d \\
& \frac{\partial \phi_{1}^{(j)}}{\partial r}=i k_{0} G\left[\phi_{1}^{(j)}-\phi_{2}^{(j)}\right] \quad \text { on } \quad r_{j}=a_{j}, d-h \leq z \leq d
\end{aligned}
$$

Substituting Eq. (8) and (10), and using the orthogonality of depth from $z=d$ - $h$ to $d$, the first matching condition between region 1 and 2 in Eq. (13) can be rewritten as,

$$
\begin{aligned}
& \sum_{n=-\infty}^{\infty} A_{n}^{j}\left\{J_{n}\left(k_{0} a_{j}\right)-\frac{J_{n}^{\prime}\left(k_{0} b_{j}\right)}{Y_{n}^{\prime}\left(k_{0} b_{j}\right)} Y_{n}\left(k_{0} a_{j}\right)\right\} \int_{d-h}^{d}\left\{\frac{\cosh \left\{k_{0}(z-d+h)\right\}}{\cosh \left(k_{0} h\right)}\right\}^{2} d z \\
& =\sum_{n=-\infty}^{\infty}\left[+e_{n}^{j} \frac{H_{n}\left(k a_{j}\right)}{H_{n}^{\prime}\left(k a_{j}\right)}+\sum_{\substack{l=1 \\
l \neq 1}}^{N} \sum_{m=-\infty}^{m=\infty} C_{m}^{l} \frac{a_{l} J_{n}\left(k a_{j}\right)}{a_{j} H_{m}(\sin \beta)} J_{m}\left(k a_{j}\right) H_{m-n}\left(k R_{l j}\right) e^{i\left((m-n) \alpha_{\alpha_{j}}\right.}\right] \\
& \quad \times \int_{d-h)}^{d}\left\{\frac{\cosh k z}{\cosh k d} \frac{\cosh \left\{k_{0}(z-d+h)\right\}}{\cosh \left(k_{0} h\right)}\right\} d z
\end{aligned}
$$

Applying the orthogonal property to the second matching conditions in Eq. (13), with respect to $z$ over the region of validity, the following equation can be obtained:

$$
\int_{0}^{d} \frac{\partial \phi_{2}^{j}}{\partial r}\left\{\frac{\cosh k z}{\cos k d}\right\} d z=\int_{0}^{d-h} \frac{\partial \phi_{2}^{j}}{\partial r} \frac{\cosh k z}{\cos k d} d z+\int_{d-h}^{d} \frac{\partial \phi_{1}^{j}}{\partial r} \frac{\cosh k z}{\cos k d} d z
$$

By applying Eq. (14) to Eq. (15), the key equation for unknown coefficients $C_{n}^{j}$ can be obtained as follows,

$$
\left|\begin{array}{l}
k \int_{0}^{d}\left\{\frac{\cosh k z}{\cosh k d}\right\}_{n}^{j} d z-\frac{H_{n}\left(k a_{j}\right)}{H_{n}^{\prime}\left(k a_{j}\right)} \\
\times \frac{\left\{J_{n}^{\prime}\left(k_{0} a_{j}\right)-\frac{J_{n}^{\prime}\left(k_{0} b_{j}\right)}{Y_{n}^{\prime}\left(k_{0} b_{j}\right)} Y_{n}^{\prime}\left(k_{0} a_{j}\right)\right\}_{0}}{\left\{J_{n}\left(k_{0} a_{j}\right)-\frac{J_{n}^{\prime}\left(k_{0} b_{j}\right)}{Y_{n}^{\prime}\left(k_{0} b_{j}\right)} Y_{n}\left(k_{0} a_{j}\right)\right\}+\frac{i}{G}\left\{J_{n}^{\prime}\left(k_{0} a_{j}\right)-\frac{J_{n}^{\prime}\left(k_{0} b_{j}\right)}{Y_{n}^{\prime}\left(k_{0} b_{j}\right)} Y_{n}^{\prime}\left(k_{0} a_{j}\right)\right\}} \\
\times \frac{\int_{d-h}^{d}\left\{\frac{\cosh k_{0}(z-d+h)}{\cosh k_{0} h} \frac{\cosh k z}{\cosh k d}\right\} d z \times \int_{d-h}^{d}\left\{\frac{\cosh k_{0}(z-d+h)}{\cosh k_{0} h} \frac{\cosh k z}{\cosh k d}\right\} d z}{\int_{d-h}^{d}\left\{\frac{\cosh k_{0}(z-d+h)}{\cosh k_{0} h}\right\}^{2} d z}
\end{array}\right|
$$$$
+\sum_{l=1}^{N} \sum_{m=-\infty}^{m=\infty} C_{m}^{l} \frac{a_{t} H_{m-n}\left(k R_{l j}\right)}{a_{j} H_{m}{ }^{\prime}\left(k a_{l}\right)} e^{i(m-n) \alpha_{i j}} \times J_{n}^{\prime}\left(k a_{j}\right) k \int_{0}^{d}\left\{\frac{\cosh k z}{\cosh k d}\right\}^{2} d z
$$$$
-\sum_{\substack{l=1 \\ l=j}}^{N} \sum_{m=-\infty}^{m=\infty} C_{m}^{l} \frac{a_{l} H_{m-n}\left(k R_{l j}\right)}{a_{j} H_{m}{ }^{\prime}\left(k a_{l}\right)} e^{i(m-n) \alpha_{i j}} \times J_{n}\left(k a_{j}\right)
$$$$
\times\left[\frac{\left\{J_{n}^{\prime}\left(k_{0} a_{j}\right)-\frac{J_{n}^{\prime}\left(k_{0} b_{j}\right)}{Y_{n}^{\prime}\left(k_{0} b_{j}\right)} Y_{n}^{\prime}\left(k_{0} a_{j}\right)\right\} k_{0}}{\left\{J_{n}\left(k_{0} a_{j}\right)-\frac{J_{n}^{\prime}\left(k_{0} b_{j}\right)}{Y_{n}^{\prime}\left(k_{0} b_{j}\right)} Y_{n}\left(k_{0} a_{j}\right)\right\}+\frac{i}{G}\left\{J_{n}^{\prime}\left(k_{0} a_{j}\right)-\frac{J_{n}^{\prime}\left(k_{0} b_{j}\right)}{Y_{n}^{\prime}\left(k_{0} b_{j}\right)} Y_{n}^{\prime}\left(k_{0} a_{j}\right)\right\}}\right]
$$$$
\times\left\lfloor\frac{\int_{d-h}^{d}\left\{\frac{\cosh k_{0}(z-d+h)}{\cosh k_{0} h} \frac{\cosh k z}{\cosh k d}\right\} d z \times \int_{d-h}^{d}\left\{\frac{\cosh k_{0}(z-d+h)}{\cosh k_{0} h} \frac{\cosh k z}{\cosh k d}\right\} d z}{\int_{d-h}^{d}\left\{\frac{\cosh k_{0}(z-d+h)}{\cosh k_{0} h}\right\} d z}\right\rfloor
$$$$
=-e^{i k\left(x_{j} \cos \beta+y_{j} \sin \beta\right)} e^{i n(\pi / 2-\beta)}
$$$$
\left[\begin{array}{l}
J_{n}^{\prime}\left(k a_{j}\right) k \int_{0}^{d}\left\{\frac{\cosh k z}{\cosh k d}\right\}^{2} d z-J_{n}\left(k a_{j}\right) \\
\times \frac{\left\{J_{n}^{\prime}\left(k_{0} a_{j}\right)-\frac{J_{n}^{\prime}\left(k_{0} b_{j}\right)}{Y_{n}^{\prime}\left(k_{0} b_{j}\right)} Y_{n}^{\prime}\left(k_{0} a_{j}\right)\right\}_{0}}{\left\{J_{0}\left(k_{0} a_{j}\right)-\frac{J_{n}^{\prime}\left(k_{0} b_{j}\right)}{Y_{n}^{\prime}\left(k_{0} b_{j}\right)} Y_{n}\left(k_{0} a_{j}\right)\right\}+\frac{i}{G}\left\{J_{n}^{\prime}\left(k_{0} a_{j}\right)-\frac{J_{n}^{\prime}\left(k_{0} b_{j}\right)}{Y_{n}^{\prime}\left(k_{0} b_{j}\right)} Y_{n}^{\prime}\left(k_{0} a_{j}\right)\right\}} \\
\int_{d-h}^{d}\left\{\frac{\cosh k_{0}(z-d+h)}{\cosh k_{0} h} \frac{\cosh k z}{\cosh k d}\right\} d z \times \int_{d-h}^{d}\left\{\frac{\cosh k_{0}(z-d+h)}{\cosh k_{0} h} \frac{\cosh k z}{\cosh k d}\right\} d z \\
\int_{d-h}^{d}\left\{\frac{\cosh k_{0}(z-d+h)}{\cosh k_{0} h}\right\}^{2} d z
\end{array}\right]
$$

In order to calculate the potential coefficients from the infinite system, Eq. (16) is truncated to $(2 M+1) N$ equations with $(2 M+1) N$ unknown values for $j=1,2, \ldots, N$ and $n=-M, \ldots$, $M$. 


$$
\begin{aligned}
& C_{n}^{j}\left\{k S_{1}-\frac{H_{n}\left(k a_{j}\right)}{H_{n}{ }^{\prime}\left(k a_{j}\right)} \frac{Q_{1} k_{0}}{Q_{3}} \frac{S_{2}}{S_{3}}\right\} \\
& +\sum_{\substack{l=1 \\
l=j}}^{N} \sum_{m=-M}^{m=M} C_{m}^{\prime} \frac{a_{l} H_{m-n}\left(k R_{l j}\right)}{a_{j} H_{m}{ }^{\prime}\left(k a_{l}\right)} e^{i(m-n) \alpha_{\phi_{j}}}\left\{\begin{array}{l}
J_{n}^{\prime}\left(k a_{j}\right) k S_{1} \\
-J_{n}\left(k a_{j}\right) \frac{Q_{1} k_{0}}{Q_{3}} \frac{S_{2}}{S_{3}}
\end{array}\right\} \\
& =-e^{i k\left(x_{j} \cos \beta+y_{j} \sin \beta\right)} e^{i n(\pi / 2-\beta)}\left\{\begin{array}{l}
J_{n}^{\prime}\left(k a_{j}\right) k S_{1} \\
-J_{n}\left(k a_{j}\right) \frac{Q_{1} k_{0}}{Q_{3}} \frac{S_{2}}{S_{3}}
\end{array}\right\}
\end{aligned}
$$

where,

$$
\begin{aligned}
& Q_{1}=\left\{J_{n}^{\prime}\left(k_{0} a_{j}\right)-\frac{J_{n}^{\prime}\left(k_{0} b_{j}\right)}{Y_{n}^{\prime}\left(k_{0} b_{j}\right)} Y_{n}^{\prime}\left(k_{0} a_{j}\right)\right\} \\
& Q_{2}=\left\{J_{n}\left(k_{0} a_{j}\right)-\frac{J_{n}^{\prime}\left(k_{0} b_{j}\right)}{Y_{n}^{\prime}\left(k_{0} b_{j}\right)} Y_{n}\left(k_{0} a_{j}\right)\right\} \\
& Q_{3}=Q_{2}+\frac{i}{G} Q_{1}, S_{1}=\int_{0}^{d}\left\{\frac{\cosh k z}{\cosh k d}\right\}^{2} d z \\
& S_{2}=\int_{d-h}^{d}\left\{\frac{\cosh k_{0}(z-d+h)}{\cosh k_{0} h} \frac{\cosh k z}{\cosh k d}\right\} d z \times \int_{d-h}^{d}\left\{\frac{\cosh k_{0}(z-d+h)}{\cosh k_{0} h} \frac{\cosh k z}{\cosh k d}\right\} d z \\
& S_{3}=\int_{d-h}^{d}\left\{\frac{\cosh k_{0}(z-d+h)}{\cosh k_{0} h}\right\}^{2} d z
\end{aligned}
$$

By using a stand matrix technique, the equations on $C_{n}^{j}$ can be solved and the unknown coefficients $A_{n}^{j}$ may then be obtain from Eq. (14) by applying $C_{n}^{j}$. In this manner the velocity potential in each fluid region $\left(\phi_{1}^{j}, \phi_{2}^{j}\right)$ can be determined.

After solving the velocity potentials, the wave excitation forces on each dual cylindrical cylinder are obtained using the integration of pressure on the wetted surface of cylinder. Wave forces in $x$-direction $\left(F_{x}\right)$ and in $y$-direction $\left(F_{y}\right)$ are calculated as follows,

$$
\begin{aligned}
& F_{x}^{j}=-i \rho \omega \int_{d-h}^{d} \int_{0}^{2 \pi} \frac{-i g H}{2 \omega}\left\{\phi_{2}^{j}-\phi_{1}^{j}\right\} a_{j} \cos \theta d \theta d z \\
& F_{y}^{j}=-i \rho \omega \int_{d-h}^{d} \int_{0}^{2 \pi} \frac{-i g H}{2 \omega}\left\{\phi_{2}^{j}-\phi_{1}^{j}\right\} a_{j} \sin \theta d \theta d z \\
& \text { on } \quad d-h \leq z \leq d \\
& F_{x}^{j}=-i \rho \omega \int_{d-h}^{d} \int_{0}^{2 \pi} \frac{-i g H}{2 \omega}\left\{\phi_{1}^{j}\right\} b_{j} \cos \theta d \theta d z \\
& F_{y}^{j}=-i \rho \omega \int_{d-h}^{d} \int_{0}^{2 \pi} \frac{-i g H}{2 \omega}\left\{\phi_{1}^{j}\right\} b_{j} \sin \theta d \theta d z \\
& \text { on } \quad d-h \leq z \leq d \\
& F_{x}^{j}=-i \rho \omega \int_{0}^{d-h} \int_{0}^{2 \pi} \frac{-i g H}{2 \omega}\left\{\phi_{2}^{j}\right\} a_{j} \cos \theta d \theta d z \\
& F_{y}^{j}=-i \rho \omega \int_{0}^{d-h} \int_{0}^{2 \pi} \frac{-i g H}{2 \omega}\left\{\phi_{2}^{j}\right\} a_{j} \sin \theta d \theta d z \\
& \text { on } \quad 0 \leq z \leq d-h
\end{aligned}
$$

where Eq. (18) is for porous part and Eq. (19) is for impermeable portion of dual cylindrical cylinder.

\section{NUMERICAL RESULTS AND DISCUSSION}

Fig. 2 shows the comparison of numerical results between the present method and the Linton and Evans [5] to examine the wave force interaction acting on the array of impermeable cylindrical cylinders. The various parameters are $a / d=1 / 2$,
$S / d=2$ and $\beta=\pi / 4$. The cylinders are numbered clockwise from 1 to 4 , and are situated at $(-2 \mathrm{a}, 2 \mathrm{a}),(2 \mathrm{a}, 2 \mathrm{a}),(2 \mathrm{a},-2 \mathrm{a})$ and $(-2 a,-2 a)$ respectively, so that the wave forces in the direction of wave advance on cylinders 1 and 3 are identical. It is understood that interaction effects can be significantly important in determining the amplitude of the wave forces. It is noted that the wave force by the present method gives the good agreement to the results from Linton and Evans. Therefore, the present method on wave force evaluation is remarkably useful to evaluate the wave forces acting on the array of impermeable cylindrical cylinders.

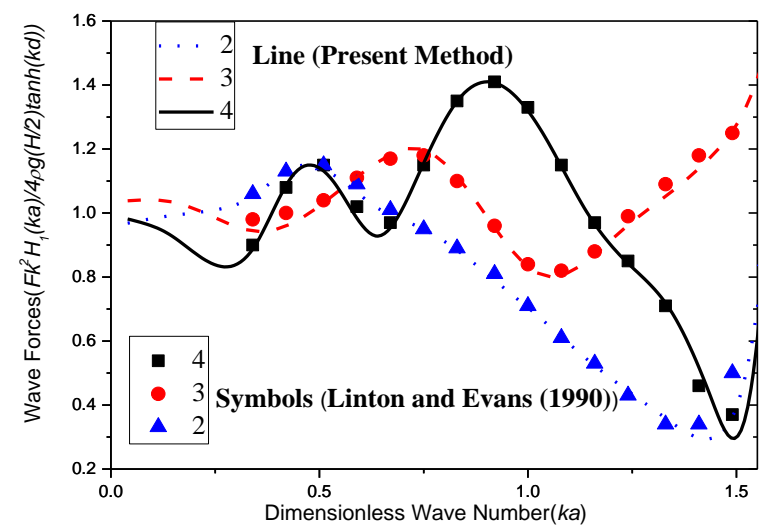

Fig. 2. Comparison of dimensional wave forces on four cylinders for $a / d=1 / 2$, $S / d=2, \beta=\pi / 4$.
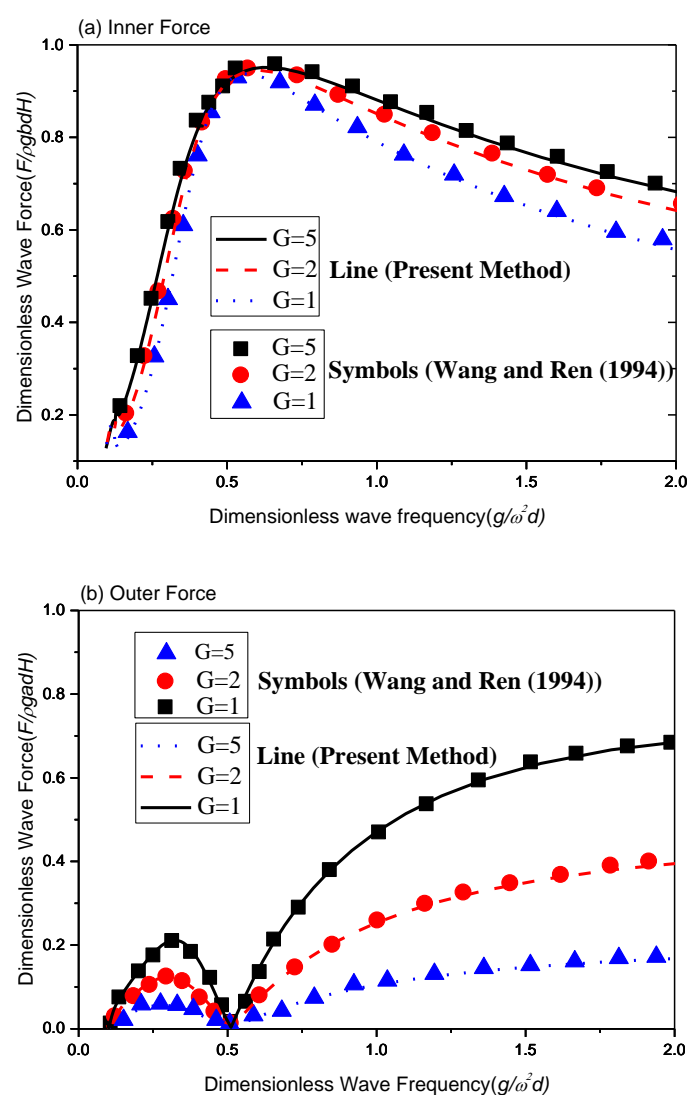

Fig. 3. Comparison of dimensionless wave forces on a dual cylindrical cylinder for $b / a=1 / 2$ and $\beta=0^{0}$ : (a) Inner force, (b) Outer force.

In order to verify the accuracy of the calculated wave forces on a dual cylindrical cylinder, which the outer cylinder is porous and the inner cylinder is impermeable, the present numerical results are compared with the numerical results of Wang and Ren [13]. It is indicated that the hydrodynamic 
force on the inner cylinder can be effectively reduced by the existence of outer porous cylinder. Fig. 3 shows the comparison of wave forces on a dual cylindrical cylinder. The various parameters are $b / a=0.5, G=1,2,5$ and $\beta=0^{0}$, respectively. The abscissa denotes the dimensionless frequency, $g / \omega^{2} d$, and the vertical axis is the dimensionless wave force normalized with $\rho g a d H$ and $\rho g b d H$, respectively. The wave force on the inner cylinder is gradually increased and the wave force on outer cylinder is inversely decreased as the porosity parameter $G$ increase. It means that the wave force on inner cylinder is increased by the wave infiltration which percolates in the inside as the porosity of outer cylinder increase. The wave force of inner cylinder has the peak value at wave frequency 0.5 ; on the other hand, the wave force of outer cylinder has the lowest value. It is indicated that the calculated wave forces are in good agreement with the results from Wang and Ren. Therefore, the present method is very available to evaluate the wave forces on the dual cylindrical structure.

In order to examine the validity of the wave force on an array of four dual cylindrical cylinders, the comparisons are made with the numerical results of Sankarbabu [20]. Fig. 4 shows the comparison of wave forces on the array of four dual cylindrical cylinders for various porosity depths $(\mathrm{h} / \mathrm{d})$ of outer cylinder. The calculation conditions are $a=5.0 \mathrm{~m}$, $b / a=0.5, d / a=5, G=1.0$ and $\beta=0.0^{\circ}$. The dual cylindrical cylinders are numbered clockwise from 1 to 4 , and situated at $(-15.0,15.0) \mathrm{m},(15.0,15.0) \mathrm{m},(15.0,-15.0) \mathrm{m}$, and $(-15.0$, $-15.0) \mathrm{m}$, respectively. The distance between cylinder centers $(S)$ is $30.0 \mathrm{~m}$, which is 6 times of cylinder radius $(S / a=6)$. In the comparison the ratio of $h / d=0.0$ indicates the full-body impermeable cylindrical cylinder, while $h / d=1.0$ represents the full-body porous cylindrical cylinder. The calculated outer and total forces are normalized by $\rho g(H / 2) a^{2}$ and the inner forces are normalized by $\rho g(H / 2) b^{2}$. Since the amount of incident wave passing through porous area is increased as the porosity depth increases, the inner force is increased. Although in the long wave region $(k a \leq 0.55)$ the pattern of inner force is strongly influenced by the porosity depth, the pattern of both porosity depth 0.5 and 1.0 becomes very similar in the short wave region $(k a \geq 0.55)$. The outer force is inversely decreased with increment of porosity depth compared to the case of inner force. The difference of outer force between the porosity depth 0.5 and 1.0 is also very small in the short wave region $(k a \geq 0.55)$. It means that the dual cylindrical cylinder with porosity depth 0.5 is very efficient to reduce the effect of wave force in the short wave region like the case of full-body porous cylindrical cylinder. The comparison of total force between the dual cylindrical cylinder with partial porous area and the full-body impermeable cylindrical cylinder $(h / d=0.0)$ is made in Fig. (c). The total forces of dual cylindrical cylinder with porosity depth 0.5 at the first and the second peaks $(k a=0.19$ and $k a=0.86$ ) decrease about $8.0 \%$ and $62.0 \%$, respectively, compared to full-body impermeable cylindrical cylinder. Especially, the dual cylindrical cylinder with partial porous area is significantly effective to reduce the effect of wave forces in the short wave region. Moreover, the calculated wave forces are in good agreement with the results from Sankarbabu in the case of full-body porous cylindrical cylinder $(h / d=1.0)$.
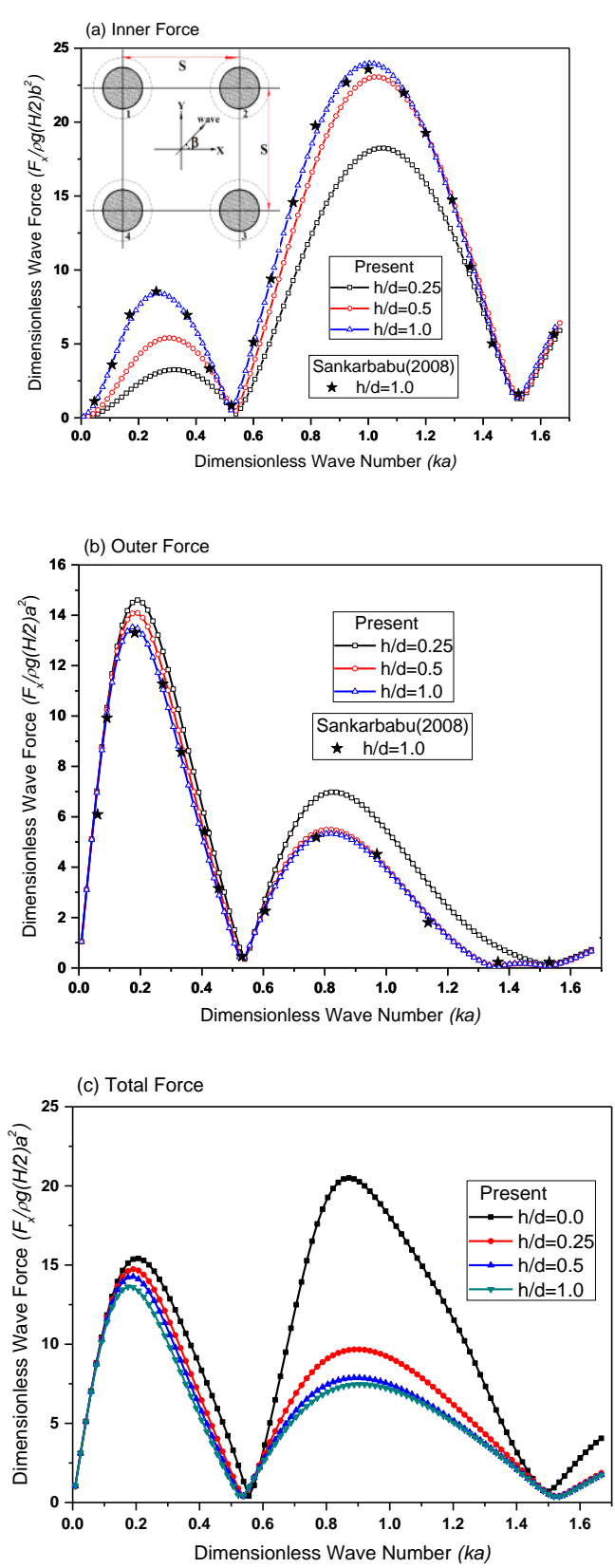

Fig. 4. Dimensionless wave forces on the array of four dual cylindrical cylinders with $a=5.0 \mathrm{~m}, b / a=0.5, S / a=6, d / a=5, G=1.0$ and $\beta=0^{\circ}$ for various porosity depths $(h / d)$ of outer cylinder: (a) Inner force, (b) Outer force, (c) Total force.

Fig. 5 shows the wave forces on the dual cylindrical cylinder with partial porous area $(h / d=0.5)$ for various porosity ratios $(G)$ of outer cylinder. Although the inner forces decrease as the porosity of outer cylinder increases, it has a very similar peak value for all cases. The outer force is significantly decreased as the porosity ratio increases and is close to zero when wave frequency is approximately 1.65 , at which the first nontrivial zero of the derivative of the Bessel function $J_{n}^{\prime}$ exists. The peak frequency of outer force is decreased as the porosity increase. It means that the structural properties of the system have been changed due to porosity of the outer cylinder. The total forces on the dual cylindrical cylinders have very similar values when the wave frequency is larger than 1.5. It means that the dual cylindrical cylinder is remarkably efficient to reduce the wave forces in the short 
wave region $(\omega \geq 1.5)$ regardless of porosity ratios.
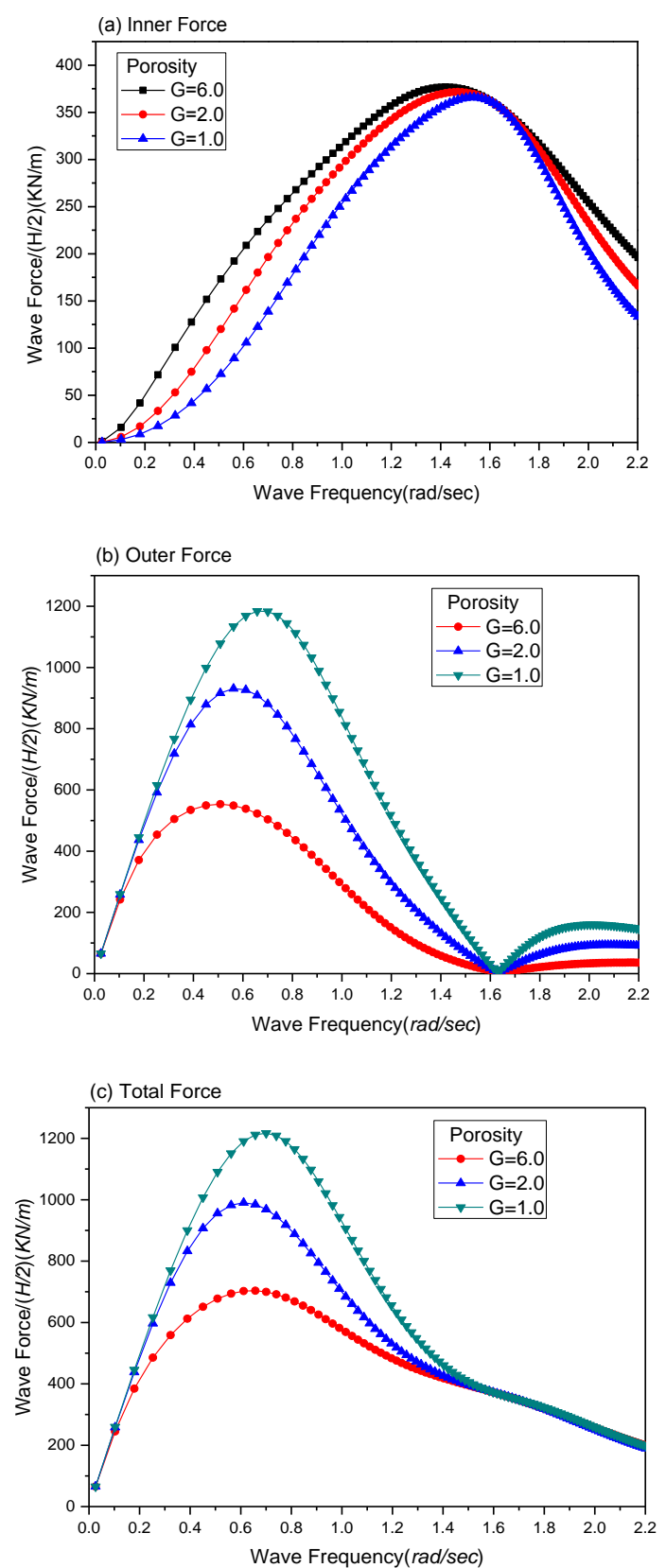

Fig. 5. Wave forces on the dual cylindrical cylinder with $a=5.0 \mathrm{~m}, b / a=0.5$, $d / a=5, h / d=0.5$ and $\beta=0^{\circ}$ for various porosity $(G)$ : (a) Inner force, (b) Outer force, (c) Total force.

Fig. 6 shows the comparison of wave run-up between the dual cylindrical cylinder and the full-body impermeable cylinder. Since the wave force has the peak value when the wave frequency is 0.7 , the comparison is made at $\omega=0.7 \mathrm{rad} / \mathrm{sec}$. The wave run-up is normalized by incident wave height $(H)$ and the abscissa denotes the angle $(\theta)$ measured counterclockwise from the positive $x$-axis. Since the incident wave energy is reduced when the incident waves pass through the porous area, the maximum outer run-up on the dual cylindrical cylinder is significantly lower than the full-body impermeable cylinder. The maximum value of outer run-up is occurred at the similar location, around the angle of $180^{\circ}$, where the incident wave propagates toward the cylinder. It is found that the dual cylindrical cylinder, when the porous-length ratio becomes a larger than $50.0 \%$ of whole draft $(h / d=0.5)$, is remarkably effective to reduce the outer wave run-up like the case of full-body porous cylinder.
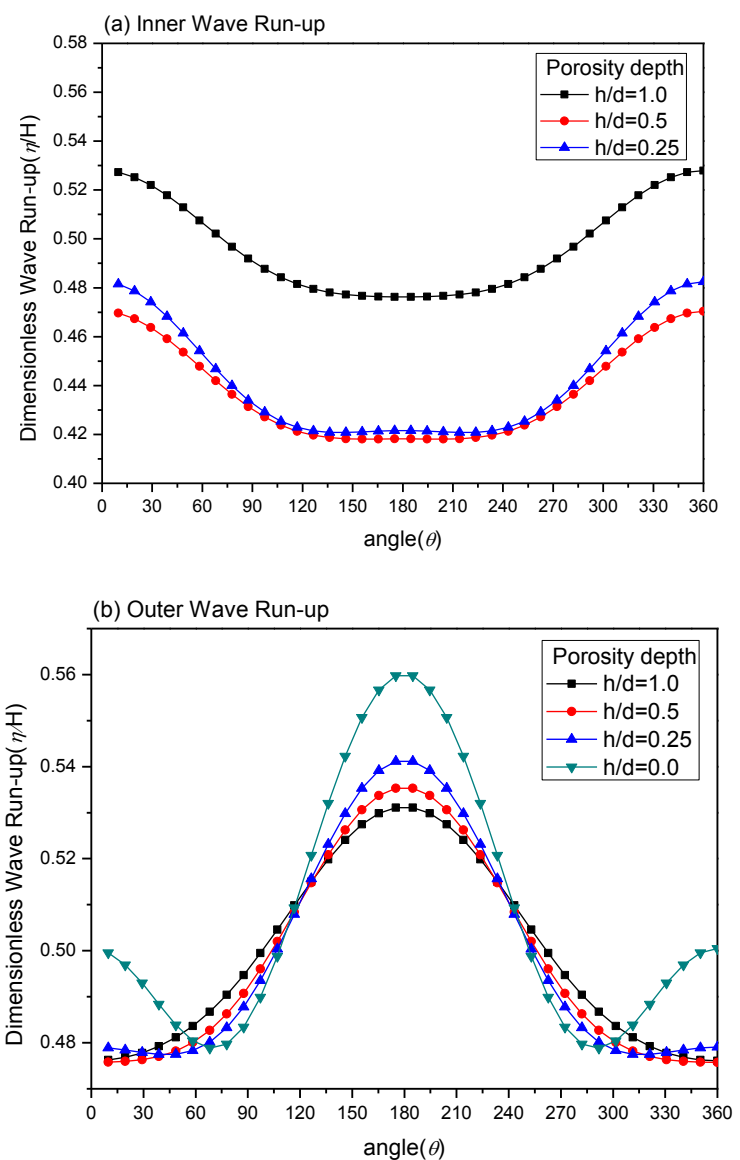

Fig. 6. Wave run-up $(\eta / H)$ on the dual cylindrical cylinder with $a=5.0 m$, $b / a=0.5, d / a=5, G=6.0, \beta=0^{\circ}$ and $\omega=0.7$ for various porosity depths $(h / d)$ of outer cylinder: (a) Inner wave run-up, (b) Outer wave run-up.

Fig. 7 shows the total force on four dual cylindrical cylinders with partial porous area for various distances $(S)$ among cylinders. The magnitude of calculated force was modulated and several peaks were observed according to wave frequency. As the gap distance changed, the location of the modulated peaks also varied. It means that wave force interaction is strongly influenced by the relationship between the incident wave length and the distance among the dual cylindrical cylinders. Although the maximum peak point on inner cylinder presents at short wave region as the distance among the dual cylindrical cylinder increase, the maximum peak point on outer cylinder appears at long wave region.

(a) Inner Force

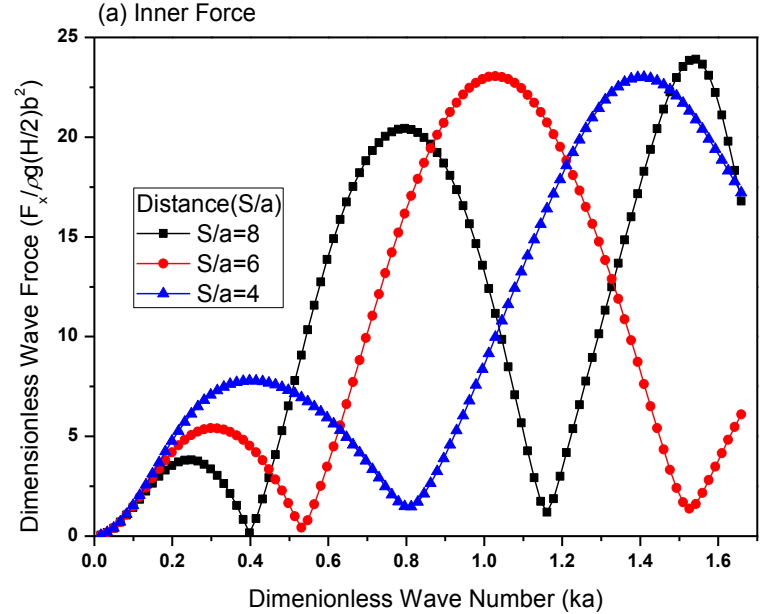



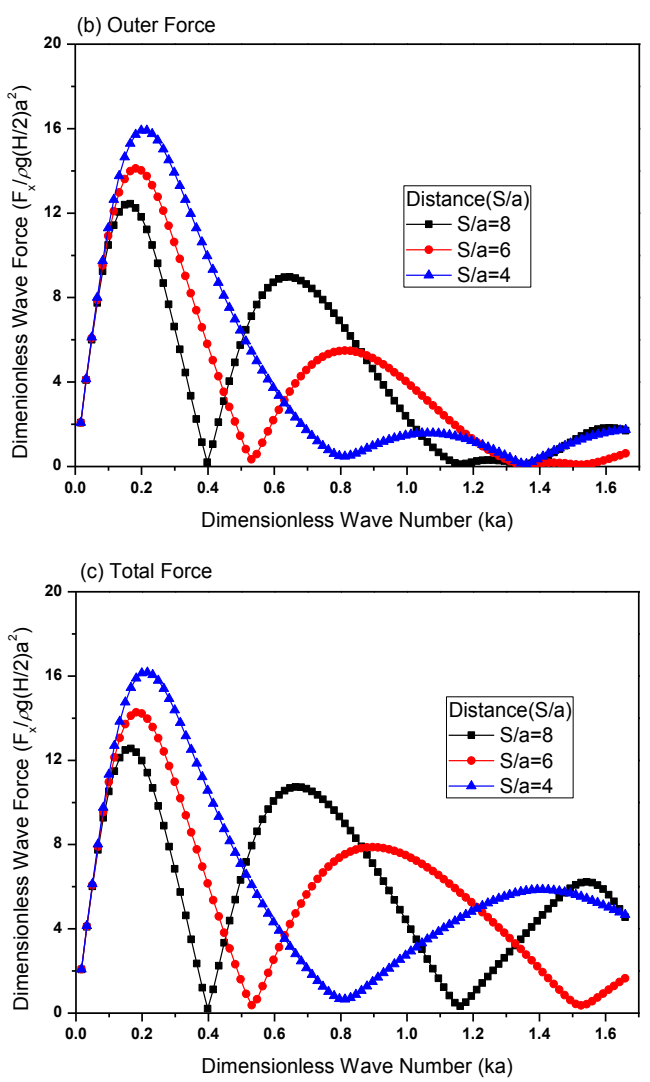

Fig. 7. Dimensionless total wave forces on the array of four dual cylindrical cylinders with $b / a=0.5, d / a=5, h / d=0.5, G=1.0, \beta=0^{0}$ for various distance (S/a) among the dual cylindrical cylinders.

\section{CONCLUSION}

Under the assumption of potential flow and linear wave theory, the 3D numerical method for the array of dual cylindrical cylinders with partial porous area is developed using the Eigenfunction expansion method and Darcy's law. For verification of this new method, the calculated results for an array of dual cylindrical cylinders are compared with the numerical results and are found to be in good agreement. It is suggested that the developed numerical method is very useful to evaluate the wave force acting on the array of dual cylinders with partial porous area, which consist of an impermeable inner cylinder and a porous outer cylinder. The water wave interaction due to the array of permeable or impermeable cylindrical cylinders is demonstrated to examine the effects of the various wave and structural parameters. The wave force caused by the interaction effects between wave and cylinders is significantly diminished as the porosity of the outer cylinder increases. It is suggested that the porosity of structure is very effective on the reduction of the wave force and wave run-up acting on the offshore structure. It is also found that a dual cylindrical cylinder with porosity depth $(h / d) 0.5$ effectively reduces the wave force like the full-body porous cylindrical cylinder. Moreover, the scattered waves from dual cylindrical cylinder with partial porous area rapidly diminish via damping as the waves pass through the porous regions.

\section{ACKNOWLEDGMENT}

This research was supported by Korea Institute of Energy Technology Evaluation and Planning through the research project "Development of $3 \mathrm{~kW}$ cylindrical wave energy system with horizontal rotation for increasing gross generation (20153030071630)" and "Development of hybrid substructure systems for offshore wind power (SUBJID0000000014637)".

\section{REFERENCES}

[1] R. C. MacCamy and R. A. Fuchs, "Wave forces on piles: A diffraction theory," Tech. Memo No. 69, US Army Corps of Engineers, Beach Erosion Board, 1954.

[2] M. S. Q. Isaacson, "Shallow wave diffraction around large cylinder," Journal of Waterway, Port, Coastal and Ocean Engineering, vol. 103, pp. 69-82, 1977.

[3] M. S. Q. Isaacson, "Wave run-up around large circular cylinder," Journal of Waterway, Port, Coastal and Ocean Engineering, vol. 104, pp. 69-79, 1978.

[4] B. H. Spring and P. L. Monkmeyer, "Interaction of plane waves with vertical cylinders," in Proc. the Fourteenth International Conference on Coastal Engineering, chap. 107, pp. 1828-1845, 1974.

[5] C. M. Linton and D. V. Evans, "The interaction of waves with arrays of vertical circular cylinder," Journal of Fluid Mechanics, vol. 215, pp. 549-569, 1990.

[6] H. Kagemoto and D. K. P. Yue, "Interactions among multiple three-dimensional bodies in water waves; an exact algebraic method," Journal of Fluid Mechanics, vol. 166, pp. 189-209, 1986.

[7] P. Mclver and D. V. Evans, "Approximation of wave forces on cylinder arrays," Applied Ocean Research, vol. 6, pp. 101-107, 1984.

[8] A. N. Williams and Z. Demirbilek, "Hydrodynamic interactions in floating cylinder arrays. Part I-wave scattering," Ocean Engineering, vol. 15 , pp. 549-582, 1988.

[9] A. N. Williams and A. G. Abul-Azm, "Hydrodynamic interactions in floating cylinder arrays, Part II-wave radiation," Ocean Engineering, vol. 16, pp. 217-264, 1989.

[10] A. N. Williams and T. Rangappa, "Approximate hydrodynamic analysis of multi-column ocean structures," Ocean Engineering, vol. 21, pp. 519-573, 1994.

[11] A. N. Williams and W. Li, "Approximate hydrodynamic analysis of multi-column ocean structures," Ocean Engineering, vol. 21, pp. 519-573, 1998.

[12] A. N. Williams and W. Li, "Water wave interaction with an array of bottom-mounted surface-piercing porous cylinder," Ocean Engineering, vol. 27, pp 841-866, 2000.

[13] K. H. Wang and X. Ren, "Interactions of cnoidal waves with cylinder arrays," Ocean Engineering, vol. 26, pp. 1-20, 1999.

[14] K. H. Wang, T. Y. Wu, and G. T. Yates, "Three-dimensional scattering of solitary waves by vertical cylinder," Journal of Waterway, Port, Coastal and Ocean Engineering, vol. 118, pp. 551-566, 1992.

[15] K. H. Wang and L. Jiang, "Interactions of solitary waves with cylinder arrays," in Proc. the International Conference on Offshore Mechanics and Arctic Engineering-OMAE, vol. 1, pp. 99-107, 1994.

[16] L. Jiang and K. H. Wang, "Hydrodynamic interactions of cnoidal waves with a vertical cylinder," Applied Ocean Research, vol. 17, pp. 277-289, 1995.

[17] K. H. Wang and X. Ren, "Water waves on a flexible and porous breakwater," Journal of Engineering Mechanics, vol. 119. pp 1025-1048, 1993.

[18] K. H. Wang and X. Ren, "Interactions of cnoidal waves with cylinder arrays," Ocean Engineering, vol. 26, pp. 1-20, 1999.

[19] M. Abramowitz and I. A. Stegun, Handbook of Mathematical Function, New York, 1972.

[20] K. Sankarbabu, S. A. Sannasiraj, and V. Sundar, "Hydrodynamic performance of a dual cylindrical caisson breakwater," Coastal Engineering, vol. 55, pp. 431-446, 2008.

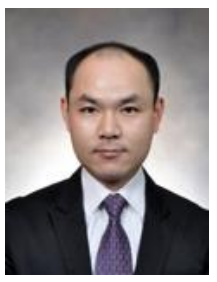

Min-Su Park received his Ph.D degree in system information engineering from Kagoshima University, Kagoshima, Japan. He has been working for Korea Institute of Civil Engineering and Building Technology (KICT) in Gyeonggi-Do, Korea since 2012 and his current position is an senior researcher. He participated in some projects for the offshore structure and offshore renewable energy. He now studies on wave energy devices and substructure for offshore wind turbine. Dr. Park is a member of KSCE (Korean Society of Civil Engineers), KSOE 
(Korean Society of Ocean Engineers), SNAK (Society of Naval Architects of Korea) and KCI (Korea Concrete Institute).

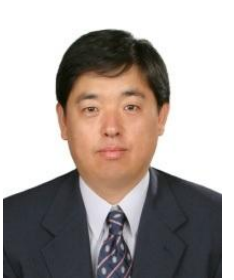

Young Jeong received his $\mathrm{Ph} . \mathrm{D}$ degree in civil engineering from Yonsei University, Seoul, Korea. He has been working for Korea Institute of Civil Engineering and Building Technology (KICT) in Gyeonggi-Do, Korea since 1994 and his current position is a research fellow. He participated in some projects for the offshore and marine structures. He now studies on offshore support structure. Dr. Jeong is a member of KSCE (Korean Society of Civil Engineers) and COSEIK (Computational Structural Engineering Institute of Korea).
Young Jun You received his Ph.D degree in civil engineering from Yonsei University, Seoul, Korea. He has been working for Korea Institute of Civil Engineering and Building Technology (KICT) in Gyeonggi-Do, Korea since 1998 and his current position is an senior researcher. He participated in some projects for developing GFRP (Glass Fiber Reinforced Polymer) reinforcement for concrete structures and strengthening and rehabilitation of structures. He now studies on offshore support structures.

Dr. You is a member of KSCE (Korean Society of Civil Engineers) and $\mathrm{KCI}$ (Korea Concrete Institute), especially was a former representative of $\mathrm{KCI}$ 